\title{
The Motor System: The Whole and its Parts
}

\author{
E. Otten \\ Department of Medical Physiology, University of Groningen, The Netherlands ${ }^{\dagger}$
}

SUMMARY

Our knowledge of components of the human motor system has been growing steadily, but our understanding of its integration into a system is lagging behind. It is suggested that a combination of measurements of forces and movements of the motor system in a functionally meaningful environment in conjunction with computer simulations of the motor system may help us in understanding motor system properties. Neurotrauma can be seen as a natural deviation, with recovery as a slow path to yet another deviant state of the motor system. In that form they may be useful in explaining the close interaction between form and function of the human motor system.

\section{KEYWORDS}

integration, virtual reality, motion capture, modeling, computer simulation

\section{INTRODUCTION}

The human motor system is exactly what the word says: a system. It displays properties that can not be understood from the components themselves. The integration of the components into a working system through the processes of phylogeny and ontogeny has happened under a strong functional guidance. Typical for the integration of biological systems is the occurrence of multi-path solutions. While in machines one particular component may provide the machine with one particular function, biological systems tend to have distributed solutions. For instance, there are often more muscles than strictly necessary to operate the motions around a joint. When one cuts more and more axons in a motor nerve of a particular muscle, the force that can be produced by the muscle does not decline gradually, but suddenly, when almost all axons are severed (Gordon et al. 1993). Lastly, when damaging a neural network more and more, its function becomes gradually diffuse. There is more interference between different aspects of the function, for instance, independent activation of muscles in a group becomes harder. However, the function of the neural network is robust in a case of minor damage. In contrast, when cutting through only one of the millions of connections in a microchip, it may be rendered useless. Many connections in a nervous system seem redundant but prove their value under trauma. This shows that the human body-machine analogy is flawed. We have learned a lot from the engineering approach to the study of the human motor system, but should be able to abandon it when it turns itself against us.

Understanding the motor system is impossible without experiments. Such experiments can be done

\footnotetext{
${ }^{\dagger}$ Antonius Deusinglaan 1, bldg 3215

P.O. Box 196, 9700 AD Groningen, The Netherlands

Fax +31 503632751

Email: e.otten@med.rug.nl
} 
on healthy human subjects and on patients with neurotrauma. In a way, neurotrauma can be seen as natural experiments by changing the anatomy of humans abruptly. Although neurotrauma is usually a horrible event, it may actually improve our insight in the intact human motor system.

In order to understand the outcome of experiments, we have to look into the architecture of the motor system itself.

\section{THE ARCHITECTURE OF THE MOTOR SYSTEM}

The human motor system consists of neural elements, muscular elements, bony elements, joints, and sensory elements. Our knowledge about the functional morphology of each of these elements is very good indeed. Neuroanatomy is a very well developed discipline and with recent staining techniques, connectivity can be traced extensively. The neurophysiology of individual neurones shows us that they are computational structures themselves. Rather than just being integrative elements for their synaptic input, they exhibit the possibility to do signal processing on the various inputs, by virtue of their complex arborisation and localized membrane properties (Van Pelt \& Uylings, 1999). Measurements of sliced preparations of the brain indicate that the processing power of neural networks is indeed as complex as may be expected from their computer simulations (Costa et al., 1999). The somatotopic mapping of the body onto the sensory and motor cortex is at first sight very well organized, resulting in the familiar homunculus pictures in textbooks, with body parts magnified in proportion to the mapped area. When looking in more detail, this organization is far from simple, resulting in highly scattered representations of one particular part of the body in several parts of the cortex (Gould et al., 1986). Moreover, the sensory representation seems to be dynamic in size, depending on the recent stimulation history of the skin of that area (Jenkins et al., 1990). This scattering has the peculiar by-effect that when some reorganization of the cortex is provoked by an amputation, sites on the still-present skin (for instance of the cheek) may me mapped on the area of amputated skin. This may give the curious experience that when crying, the tear seems to flow down the cheek, but also down the amputated arm (Ramachandran \& Blakeslee, 1998). It is very unlikely that this has some functional meaning but must be attributed to the reorganization of the noncontinuous representation of body onto the sensory cortex.

Muscle physiology as a discipline has reached a stage where there are few questions about the actual engine of force production (McMahon, 1984) and many questions about the meaning of variation between muscles (Burke et al., 1974), such as motor unit distribution. Muscle architecture in terms of functional requirements is a topic slowly gaining ground (Otten, 1988). Clearly, the available space for a muscle can be filled in many ways, but functional requirements like maximal shortening velocity and maximal force dictate how this space is filled in. On top of that, the synergy between muscles running across the same joint comes into perspective, displaying the complexity of the control that must be behind the muscle-bonetendon complex (Doorenbosch et al., 1997).

Joints keep the bony elements together while allowing for some freedom of movement relative to each other. Integrity of a joint can be defined as a state in which the limits of its motion are guarded to avoid damage. In a passive state, some of that integrity is maintained by the passive properties of the surrounding muscles. Under high energy functional conditions, the active force components of the muscles help to maintain the integrity of the joints (Gandevia, 1986).

The dynamics of multi-joint rigid body systems is very complex indeed and can be approached 
from several sides. The Newtonian approach considers a rigid body as a free body being in dynamic equilibrium in the multi-joint system. The Lagrangian approach includes the coupling of the rigid bodies by its joints into fewer equations than the Newtonian approach. The approaches should produce the same solutions. In forward dynamic simulations, a model is formulated of a system of coupled rigid bodies (like the human body) and the internal forces (like those produced by muscles), and some of the external ones are given. The simulation then calculates the resulting motion and contact forces with the external world. In this kind of simulation, one has a similar task the brain has in controlling motion: if the internal forces are ill conceived, a non functional motion results (like falling in a balance task). One thing is clear from forward dynamic simulations: The amount of kinetic energy flow along the sub-chains is extremely complex (Andrews, 1995), and any theory on the control of multiunit systems (like our own body) should include this flow in some form. An inverse dynamics simulation does exactly the opposite: it calculates the internal forces from the motion of the human body and the contact forces. This can be a very rich source of understanding because it produces functional activation patterns of muscles. From the study of movements of human beings in functional situations, like walking or throwing a ball, one can deduce that the control system includes these properties. For instance, when humans throw a ball, they decelerate the shoulder at the right time to transport kinetic energy toward the hand just before the ball is released. A major question is: how does the brain represent these complex properties while guiding the body through ill predictable mechanical conditions, such as a lumpy lawn.

Mechano-receptors are important in the motor system, since they provide the control part with vital information concerning forces, pressure, velocity of elongation, etc. The distribution of mechanoreceptors in the system suggests that it is not random. For instance muscle spindles are more abundant in parts of the muscle in which lengthening velocities are higher. The section of the masseter muscles furthest away from the jaw joint contains most of the spindles.

Spindles are very sophisticated mechanoreceptors in the sense that they have a neural control of their sensitivity. Indeed, most of the spindle properties converge on having a high sensitivity to small amounts of change in muscle length, while the optimal sensitivity can be shifted towards the used postural length (Boyd et al., 1985). This makes them an engineer's nightmare, but if one thinks about it, the spindle is almost perfectly suited to monitor muscle movements under all kinds of conditions.

\section{STUDYING THE WHOLE SYSTEM}

We may have quite a respectable knowledge about the components of the motor system, a bit about its integration, but this knowledge can only be put in its proper functional perspective by placing it in the whole functional organism. In its turn, this requires a thorough study of the human being in motion. Technology for motion capture has improved dramatically over the last few years, and it is now possible to integrate virtual reality technology with motion capture devices. Such a system has been in development over the last 2 years in a collaboration between a small company called 'Motek', the department of rehabilitation of the Amsterdam Medical Centre, and our department. The system, called CAREN, which is an acronym for 'Computer Assisted Rehabilitation Environment', consists of the following components:

a) a large video wall on which three-dimensional scenes can be shown in motion, 
b) a motion platform, controlled by a computer, that can be translated in three directions and rotated about three axes,

c) a motion capture system consisting of 6 cameras mounted to the ceiling and reflective markers affixed to the body of the subject or the patient, and

d) a human body model that can be run in real time in synchrony with the observed motion of the subject or patient.
A large software environment takes care of the data flow in real time and has been named Dflow (Fig. 1). This technology allows patients to be tested in challenging new environments, or in accurate copies of known environments, such as a city bus, taking corners etc. The purpose of this is to identify the kind of motor strategies that are used in functional situations. If there are any problems, such as those occurring in motor disorders, one can pinpoint the difference with healthy subjects.

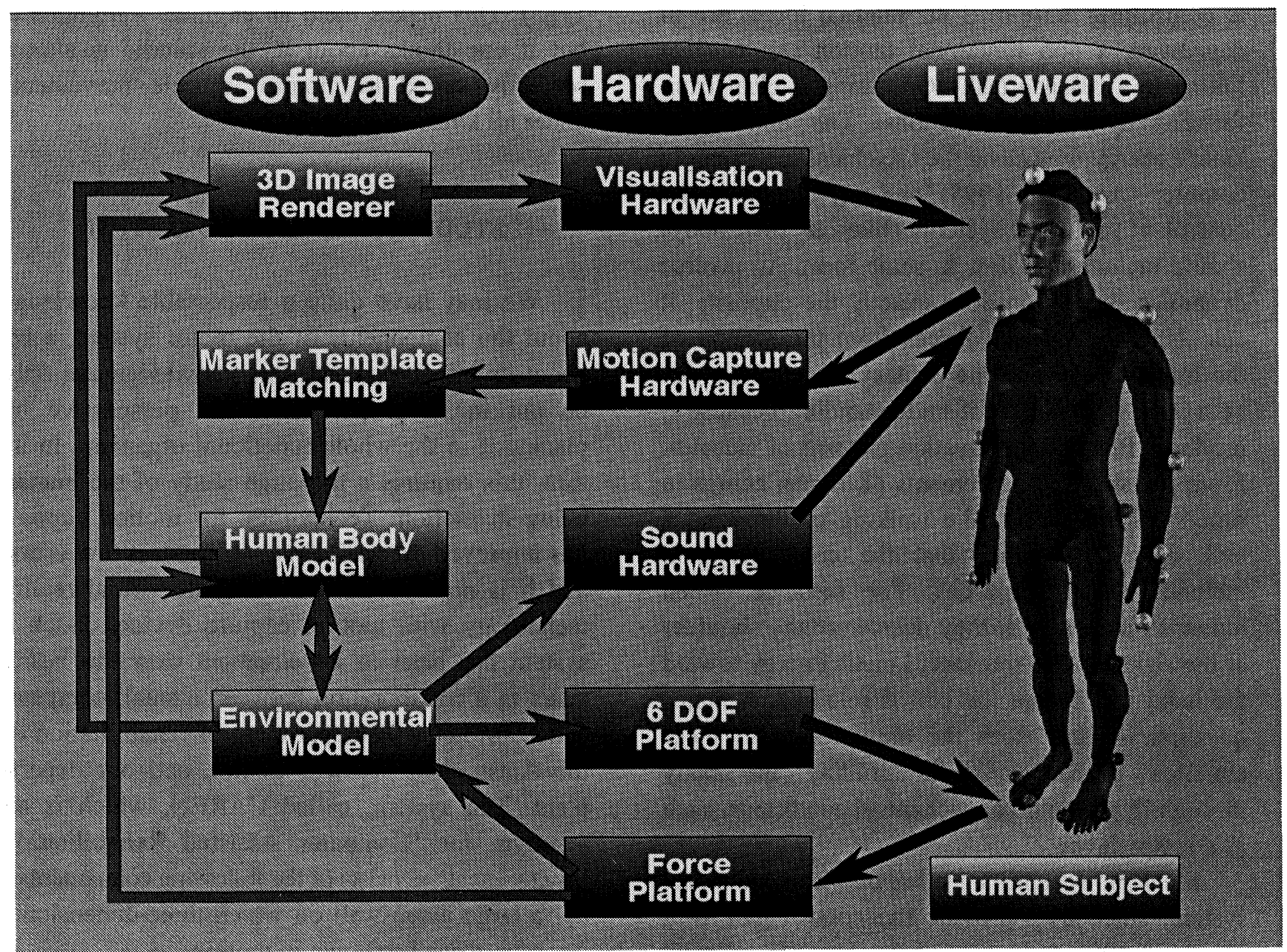

Fig. 1: Dflow software environment 
Since there has been little time since the development of the system, we have only been able to do some pilot projects. These concerned the study of patients with an amputated leg, patients with Parkinson's disease, and CVA patients.

The advantages of a virtual environment over a real environment are:

- experiments are repeatable

- sensory inputs can be separated

The first point implies that the settings of the environment can exactly be the same in experiments with different subjects or patients. For instance, a ride in a city bus can be done in exactly the same way, while a real city-bus will always have differences between rides. The second point implies that while in the real world vision and mechanics are coupled, they can be uncoupled in the virtual world. For instance, the visual flow that is associated with walking through a corridor is linked to the mechanical flow of the floor under your feet. But in the virtual world, you can create slight or gradually increasing uncoupling of these two flows. Sensorymotor integration of walking can become compromised by such an experiment, teaching us more about the synergism of the sensory inputs. CAREN is a unique new development in the field of medicine and virtual reality. However, technology itself is only like using glasses: it does not suggest any directions, it only provides sharper vision of things you direct your gaze to. And so the challenge is to bring fields of knowledge together: a biomechanical analysis of multi-joint coordination together with neuroimaging techniques and existent clinical observations. For instance, we know that after an amputation of a limb, reorganization of the sensory and motor cortex takes place. However, this only says something about the mapping in a proportional sense. The way somebody moves with an amputation means that the internal representation needs to change in a much more complex way: The bio- mechanical flow of kinetic energy changes dramatically and since so many motor tasks are performed in a feedforward mode, these new properties need to be represented centrally. The way of looking at limitations of movement control as a result of trauma can be broadened and given more depth in this way. The changes both in the periphery and centrally after neurotrauma are complex but are part of a coherent process. Explanations of what is observed should be put to the test in a functional environment, since even fundamental issues on trauma and recovery are wildly debated. The possibility to refute certain notions makes the study of motor control a true science.

\section{INTEGRATION OF DETAIL INTO THE WHOLE SYSTEM}

One of the most challenging enterprises in the study of the motor system is the integration of detailed knowledge about parts into the working whole system. As stated above, this is not easy, due to the emergence of system properties. This phenomenon has been studied by many scientists and philosophers (Dullemeijer, 1974) and is generally stated as "the whole is more than the sum of its parts". Another problem is the estimation of the importance of detailed knowledge for the understanding of the whole system. As an example:

- How important is the knowledge we have on the dynamics of cross-bridges inside skeletal muscles for the understanding of motor control as a phenomenon.

- Can we suffice with a handful of equations describing the dynamics of the whole muscle, or are molecular events, such as stiction (ongoing cross-bridge attachments after a period of constant muscle length and little activation), too important to leave out. 
Another example:

- Can we describe brain centers as boxes from which complex motor patterns flow without understanding how, or is the detailed knowledge important for the prediction of possibilities that have not been observed yet.

Just to illustrate our bad understanding of motor control as a system property, let me summarize our success with Functional Electrical Stimulation (FES) in patients with complete spinal cord transsection: none. True, the best attempts have led to something that looks like a step or two, but balanced unaided walking has never been achieved. The most successful results of FES are observed in patients with incomplete spinal cord injury as an assistance to rather than a replacement of natural motor control. Such results have been obtained with limited sets of electrode pairs (2 or 3 ) and sensors (for instance 3) (Popovic et al., 1998).

\section{THE FUTURE}

Presently we live in an exciting period in which from both sides (detail and whole) a massive amount of information is available. From clinical practice we have an almost encyclopedic layout of the various ramifications of motor disease. From the scientific world we have knowledge about the components of the motor system, as explained above. It is, however, not simple to close the gap between the two for a very simple reason rooted in the philosophy of science. As Hanson (1958) explained, our observations are guided by theoretical knowledge. The way we look at some phenomena (like the motor control of a patient in a given functional condition) is guided by what we know and expect. Observation is impossible without knowledge. Surely, the way clinical syndromes are described depends heavily on the theoretical knowledge available to the observer. Experiments in the clinical world are usually done based on a strong tradition in which this theoretical knowledge is present. Comparability can be high in repeated experiments. Even inter-observer coherence may be high. But this does not mean that the observations are directly linked to knowledge of the underlying process, the realm of basic medical science. It is exactly for this reason that I would like to promote the presence of a motor control laboratory in which the connection between clinical science and basic science is forged. Even when comparable equipment is used to measure signals, the interpretation will be richer. This does not give a guarantee that suddenly clinical observations are interpreted in the 'right' way, but it does guarantee that basic and clinical scientists learn from each other: a fusion of paradigms.

\section{NEUROTRAUMA}

Neurotrauma, caused by some mechanical or chemical process, can occur all over the nervous system. Unless the location of the trauma is very well defined and in some highway of the nervous system carrying few mixed signals, it is hard to predict the functional outcome (Curt et al., 1998). It is for that reason that observations of the combination of trauma and its functional changes can be very instructive. In practice there are a few limitations. To find the exact location and extent of the lesion is not easy, even in the light of modern scan technology. Whether transections are partial or complete can usually only be established from the functional effects. Due to the tremendous capacity of the human body to compensate for damage (which is obvious from the functional adaptations we see after trauma), we are looking not only at the direct result of the trauma but also largely to compensatory strategies. These two effects are very hard do disentangle since they are part of the same functional pattern. An example is 
trauma to a peripheral nerve, innervating the muscles of the left leg of a patient. In the walking pattern, the healthy leg shows pathological movements, which are compensations for the functional limitations of the left leg. This example, in which the location of the neurotrauma is quite easy to establish, shows that our only hope is to approach the problem from several sides. The most difficult part for our understanding is the rearrangement of the motor strategy. This rearrangement is seated in the cortex, which we don't understand very well. The biomechanical outcome, however, opens itself very well for analysis. If we perform a careful and complete 15 segment motion capture of the walking movement of the patient and perform inverse dynamics, we have the full story of the moment build up in the main joints of the patient. Comparing this with some average walking pattern of healthy subjects should reveal large deficiencies in the build up of moments in the left leg. Since the patient does not fall, a functional walking pattern exists, which means that a successful strategy has been developed as a compensation. In order to understand the deviating patterns of the moments in the joints of the right leg, and possibly in the trunk and shoulder joints, we can freeze the motion somewhere in the stepping cycle and try to insert a normal pattern of moments in the right leg. By doing forward dynamic calculation, even for a short time (like 0.1 or 0.2 seconds), it becomes evident what the effect is on the trajectory of the center of mass in space. For instance, the moments at the unimpaired side may be too weak or have the wrong combination to keep the center of mass sufficiently high, and the patient may loose height, resulting in the breakdown of the walking pattern. Slight deviations from the observed pattern of moments may be tried to establish how robust the pattern is. We know from experiments in which subjects balance a vertical stick on their hand that humans have a limited accuracy in producing the correct hand accelerations when compared with computer-controlled devices balancing a stick (Miyagawa \& Ishida, 1995). This suggests that if some pattern has been chosen, it should be robust in the face of disturbances in the moments of the magnitude of the accuracy that the patient can maintain. It is very likely that patients do not only find a solution that works but find a solution that is robust. To be more precise: they try to find a solution that is robust within the time it takes to perform further corrections on possible errors that have built up.

\section{RECOVERY FROM TRAUMA}

Recovery from trauma probably consists of two parts: a process guided by local, cellular reactions, that would have occurred in most functional states, and a process that heavily depends on the functionality in which the patient continues to live. Looking at this statement, it appears that we can learn a lot from recovery from trauma about the recovery process and the outcome but very little about motor control itself. To clarify this: the patient has moved from anatomical and physiological state $\mathbf{H}$ (for healthy) to state $\mathrm{T}$ (for trauma) in a short time. After that, the recovery leads to a state $R$ (for recovered), which is usually different from state $H$. So what we have is a sudden path from $\mathrm{H}$ to $\mathrm{T}$ and a slow one from $\mathrm{T}$ to $R$. If we express the functional abilities of a patient to be some very complex function of the present anatomical and physiological state, we have a continuously changing functional state during the slow path from $T$ to $R$. In itself, that is an interesting set of natural experiments on the functional morphology of the patient, but the exact trajectory of the path teaches us only something about the interrelation of function and recovery and nothing about motor control itself. This problem is very comparable to understanding growth. For instance the lower jaw of a child grows but does this 
not only by increasing in size but also by remodeling underway (Baumrind et al., 1997). Biomechanically speaking, there is no need to resorb bone, only to unevenly add bone in the process. Yet the resorption happens. This may be part of some ontogenetic pathway that cannot be understood from the function alone.

\section{CONCLUSION}

It is obvious: there is a huge gap between our understanding of parts of the motor system and our observations of the whole. This has its grounds in the fact that most of the properties of the whole are system properties that emerge from the integration of the components (Dullemeijer, 1974). You can spend a lifetime studying the architecture of a single muscle without being able to clarify it in terms of the use of that muscle during some functional action. From a scientific viewpoint, the gap needs to be closed: it is not sufficient to explain the observed properties of the motor system in terms that have been given meaning by those observations alone. That is not explaining, that is story telling. Moreover, a closed system of thought can defy falsifications and thus lead to tautologies (Wittgenstein, 1995).

It is proposed to close the gap by integrating our knowledge into large-scale, integrated computer simulations, fed by measurements of the motor system in functional conditions. The natural experiments of neurotrauma can be helpful, but only if exact neuroanatomical information is present of the lesion sites, possibly augmented by neurophysiological measurements.

\section{REFERENCES}

Andrews JG. 1995. Euler's and Lagrange's equations for linked rigid-body models of three-dimensional human motion. In: Allard, P., Stokes, IAF, Blanchi, JP, eds, Three-Dimensional Analysis of Human
Movement. Champaign, Illinois, USA: Human Kinetics.

Baumrind S, Bravo LA, Ben-Bassat Y, Curry S, Korn EL. 1997. Lower molar and incisor displacement associated with mandibular remodeling, AngleOrthod 67: 93-102.

Boyd IA, Murphy PR, Moss VA. 1985. Analysis of primary and secondary afferent responses to stretch during activation of the dynamic bag1 fibre or the static bag2 fibre in cat muscle spindles. In: Boyd IA, Gladden MH, eds, The Muscle Spindle. London, UK: MacMillan Press.

Burke RE, Levine DN, Salcman M, Tsairis P. 1974. Motor units in cat soleus muscle: Physiological, histochemical and morphological characteristics. J Physiol 238: 503-514.

Costa L da F, Cesar RM Jr, Coelho RC, Tanaka JS. 1999. Analysis and synthesis of morphologically realistic neural networks. In: Poznanski RR, ed, Modeling in the Neurosciences. Amsterdam, The Netherlands: Harwood Academic Publ., 505-529.

Curt A, Keck ME, Dietz V. 1998. Functional outcome following spinal cord injury: Significance of motor-evoked potentials and ASIA scores. Arch Phys Med Rehab 79: 81-86.

Doorenbosch CA, Welter TG, van-Ingen-Schenau GJ. 1997. Intermuscular coordination during fast contact control leg tasks in man. Brain-Res 751: 239-446.

Dullemeijer P. 1974. Concepts and approaches in animal morphology. Assen, the Netherands: Van Gorcum \& Comp. B.V.

Gandevia SC. 1986. Somatosensory activity relevant for motor output. A summary of presentations and discussion. Appl Neurophysiol 49: 241-250.

Gordon T, Yang JF, Ayer K, Stein RB, Tyreman N. 1993. Recovery potential of muscle after partial denervation: A comparison between rats and humans. Brain Res Bull 30: 477-482.

Gould HJ, Cusick CG, Pons TP, Kaas JH. 1986. The relationship of corpus callosum connections to electrical stimulation maps of motor, supplementary motor, and the frontal eye fields in owl monkeys. J Comp Neurol 247: 297-325.

Hanson NR. 1958. Patterns of Discovery. Cambridge, UK: Cambridge University Press.

Jenkins WM, Merzenich MM, Ochs MT, Allard T, Guic-Robles E. 1990. Functional reorganization of primary somatosensory cortex in adult owl 
monkeys after behaviorally controlled tactile stimulation. J Neurophysiol 63: 82-104.

McMahon Th.A. 1984. Muscles, Reflexes and Locomotion. New Jersey, USA: Princeton University Press.

Miyagawa T, Ishida Y. 1995. Neural Network-Based Model Reference Control for Inverted Pendulum. Proceedings ICNN; 640

Otten E. 1988. Concepts and models of functional architecture in skeletal muscle. In: Pandolf KB, ed, Exercise and Sport Science Reviews, New York, NY, USA: MacMillan; 16: 89-139.
Pelt J van, Uylings HBM. 1999. Natural variability in the geometry of dendritic branching patterns. In: Poznanski RR, ed, Modeling in the Neurosciences. Amsterdam, the Netherlands: Harwood Academic Publishers; 79-109.

Popovic, MR, Keller, T, Morari, M, Dietz, V. 1998. Neural prostheses for spinal cord injured subjects. J BioWorld 1: 6-9.

Ramachandran, VS, Blakeslee S. 1998. Phantoms in the Brain. New York, NY, USA: William Morrow.

Wittgenstein L. 1995. Tractatus Logico-Philosophicus. London:, UK: Routledge Kegan \& Paul. 

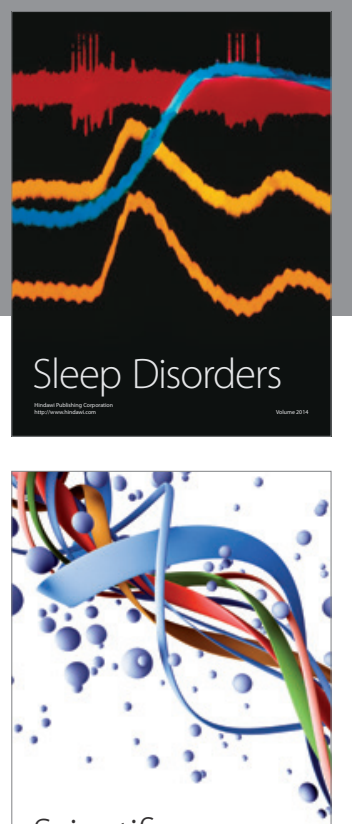

Scientifica
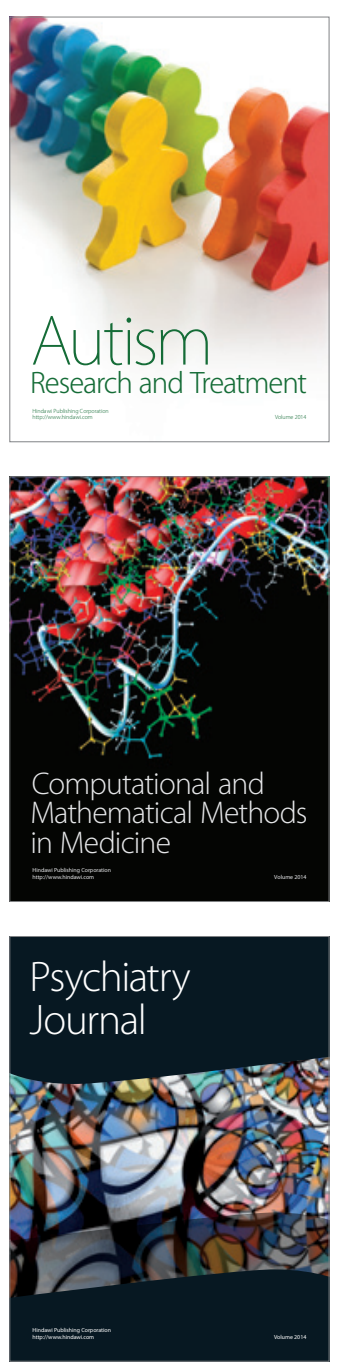
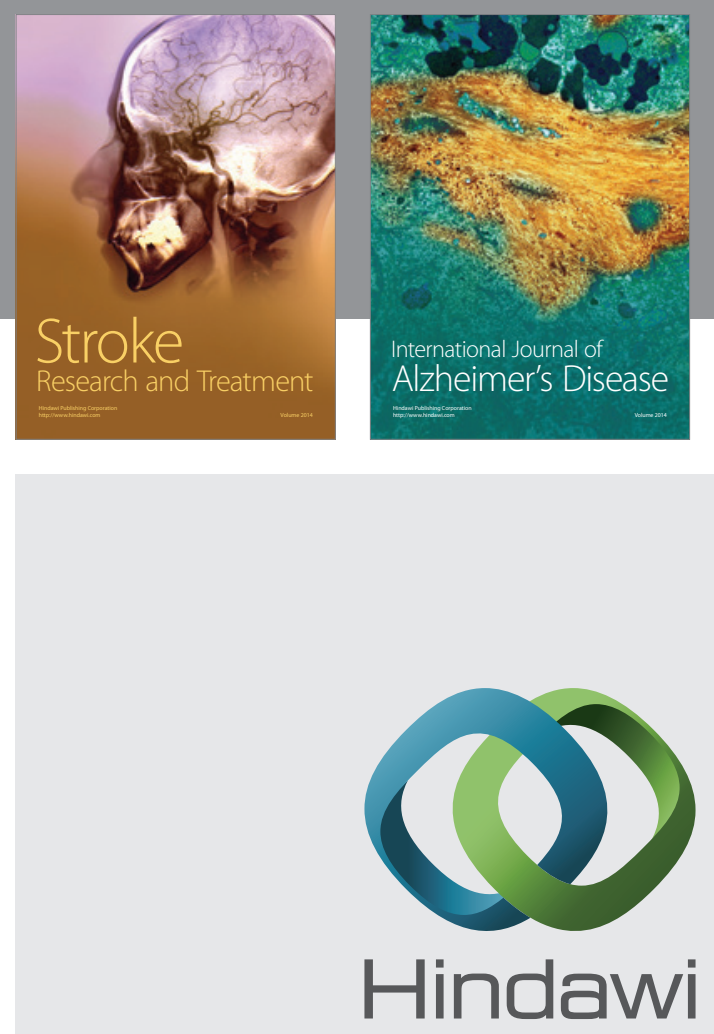

Submit your manuscripts at

http://www.hindawi.com
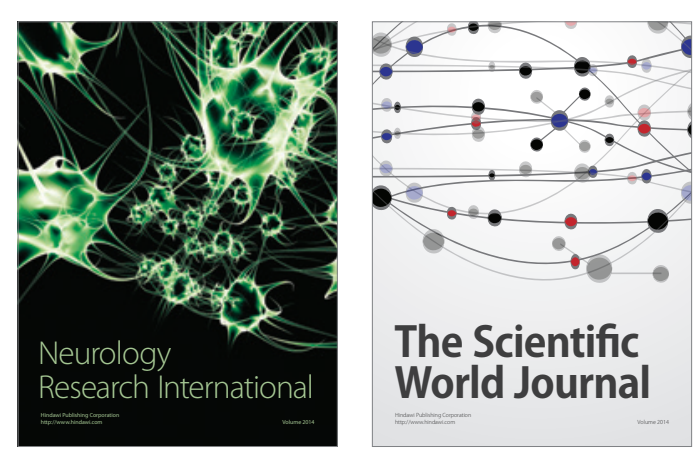

The Scientific World Journal

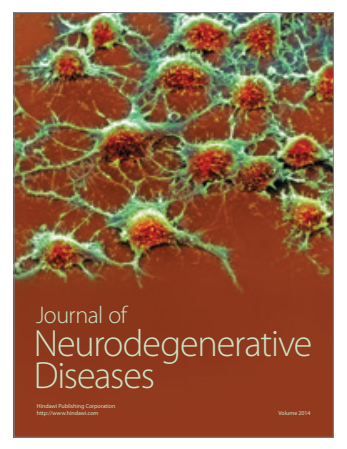

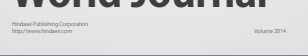

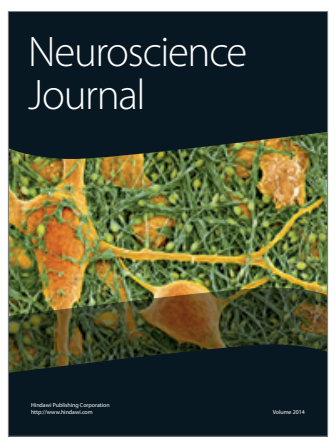

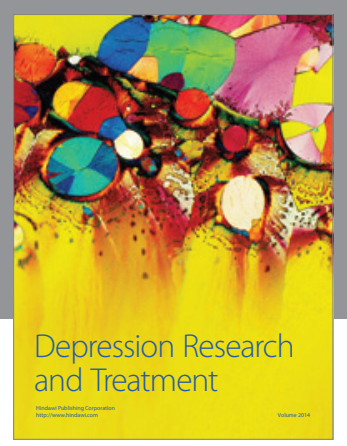
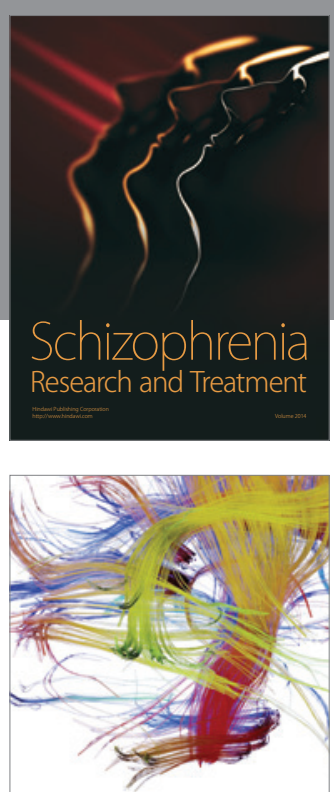

Brain Science

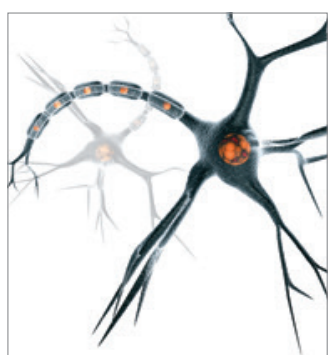

Neural Plasticity
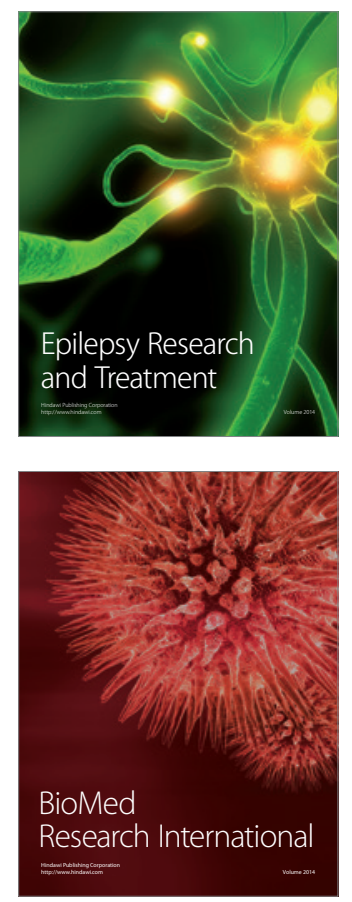

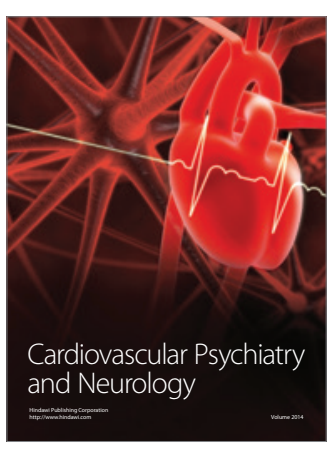

Parkinson's

Disease
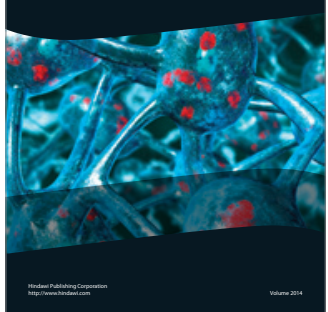